\title{
EMERGENCY MEDICINE POINT OF VIEW ON THE EPIDEMIOLOGY OF DIABETES AND DIABETES-RELATED COMPLICATIONS
}

\author{
Patrycja Szalast $\odot$, Lukasz Szarpak $\odot$
}

Lazarski University, Warsaw, Poland

\begin{abstract}
INTRODUCTION: Despite centuries of research and clinical work, the diagnosis of diabetes was still treated as a quick death sentence at the beginning of the 20th century. Unfortunately, the number of patients with diabetes is increasing every year. Type 2 diabetes is increasingly common among young people: children and adolescents. The article discusses the type of diabetes and the importance of rapid diagnosis and management. Type 1 diabetes, type 2 diabetes, latent autoimmune diabetes in adults, monogenic diabetes, gestational diabetes were analysed. Life-threatening conditions resulting from complications of diabetes including diabetic ketoacidosis, hyperglycaemic-hyperosmolar state, hypoglycaemia, lactic acidosis were discussed, and the first-line emergency treatment were analysed. The life-threatening complications of diabetes and rescue procedures in these conditions were discussed in detail.
\end{abstract}

CONCLUSIONS: Due to the frequency of life-threatening complications, diabetes is a heavy burden on the medical staff. Adequate diagnosis and implementation of appropriate treatment significantly improve the patient's condition.

KEY WORDS: diabetes; emergency medicine; treatment; epidemiology; diagnosis

Disaster Emerg Med J 2020; 5(1): 41-48

\section{INTRODUCTION}

Diabetes has been a disease known to people for at least five thousand years. The oldest preserved description of diabetes, today called type 1 diabetes, is contained in the Egyptian papyrus dating back to around 1550 BC. Areteus of Cappadocia was the first physician to use the term "diabetes" in his work "Acute and chronic diseases" [1].

Despite centuries of research and clinical work, the diagnosis of diabetes was still treated as a quick death sentence at the beginning of the 20th century. The prognosis for diabetic patients improved after the introduction of insulin to medicine in 1922, followed by oral anti-diabetic drugs.
Unfortunately, the number of patients with diabetes is increasing every year. It is estimated that in 2014, more than 422 million adults worldwide suffered from diabetes. In the last decade, the prevalence of diabetes has increased faster in lowand middle-income countries than in high-income countries. In 2012, diabetes caused 1.5 million deaths. Higher than optimal blood glucose levels caused an additional 2.2 million deaths, increasing the risk of cardiovascular and other diseases [2].

Diabetes is an increasingly common disease. In Poland, it affects nearly 3 million people, $80 \%$ of whom are patients with type 2 diabetes. Over 5 million Poles have a pre-diagnosed pre-diabetes condi- 
tion. Unhealthy dietary habits and lack of physical activity cause that the number of patients is constantly growing. Unfortunately, type 2 diabetes is increasingly common among young people: children and adolescents. Diabetes is the first non-infectious disease recognized more than 10 years ago by the United Nations as an epidemic of the XXI century [3].

\section{Type 1 diabetes}

\section{TYPES OF DIABETES}

Contrary to popular belief, type 1 diabetes is not a child-age disease. It occurs at all ages, in people of all races [2]. Depends on the rate of $\beta$ cell loss. Rapid exhaustion of $\beta$ cell secretion reserves in children and adolescents is the cause of sudden onset of the disease (the first symptoms of which are acidosis and ketosis) and unstable course of diabetes mellitus with tendency to glucose fluctuations from hyperglycaemia to hypoglycaemic coma. The fluctuating course of diabetes accelerates the development of chronic complications, which may appear as early as from the 5th year of the disease's duration [3].

\section{Latent Autoimmune Diabetes in Adults}

The definition of type 1 diabetes with autoimmune aetiology includes autoregressive, slowly developing diabetes. Latent Autoimmune Diabetes in Adults (LADA) is a late manifestation of autoimmune diabetes in adults, most commonly diagnosed in patients over 35 years of age, characterized by clinical insulin dependence in the first months after diagnosis [4]. Type LADA belongs to type 1 diabetes with a slow progressing autoimmune process of $\beta$-cell destruction. This subtype of diabetes affects $5-10 \%$ of people with diabetes diagnosed as type 2 diabetes after the age of 35 . Clinical symptoms in LADA type diabetes do not always allow to make the final diagnosis, causing diagnostic difficulties in differentiation with type 2 diabetes [5].

For a reliable diagnosis of LADA diabetes, the presence of antibodies typical of type 1 diabetes, in particular, anti-GAD65, and/or a low $C$ peptide concentration is necessary $[6,7]$.

In adults, at the time of diagnosis of type 1 diabetes, the following features are most common:

- the age of 20-50 years;

- slim figure;

- no diabetes in the family;

- increased clinical symptoms of hyperglycaemia (polyuria, increased thirst);
- ketoacidosis or ketonuria;

- significant (5-10 kg) unintentional weight loss within a few weeks;

- normal urine sediment pattern;

- a moderately elevated $\mathrm{HbA}_{1 c}$ value at the time of diagnosis.

Once the glycaemia is stabilized in these patients, lipid metabolism and arterial blood pressure are usually normal. It should be remembered that routine autoimmune tests and serum C-peptide testing are not necessary to diagnose type 1 diabetes. The diagnosis of classical symptoms of hyperglycaemia and the above-mentioned features, as well as the presence of ketonuria, justify the diagnosis of type 1 diabetes mellitus and consequently the initiation of insulin therapy. In doubtful cases, the determination of islet cell antibody (ICA) and autoantibodies to glutamic acid decarboxylase (anti-GAD) makes it very easy to determine the correct diagnosis [8]. Even a slight increase in any of the above antibody parameters suggests an autoimmune background of hyperglycaemia. C-peptide assessment, especially only in fasting conditions, is of no significance for differentiation of diabetes types or prognosis of treatment course [1].

\section{Monogenic diabetes}

It is conditioned by the mutation of a single gene, leading in most cases to a defect in insulin secretion and represents $1-2 \%$ of all cases of diabetes. The final diagnosis is established on the basis of the result of a genetic test [9]. The importance of diagnosing monogenic diabetes results from the possibility of family prognosis [10]. The most common forms of monogenic diabetes are Maturity Onset Diabetes of the Young (MODY), mitochondrial diabetes and neonatal diabetes [11].

\section{Type 2 diabetes}

Diabetes type 2 is a complex and multi-factorial disease that occurs through the interaction of genetic and environmental factors leading to two major overlapping pathologies: a defect in insulin secretion and a reduction in insulin sensitivity in peripheral tissues [12].

The development of the disease begins with a gradual increase in insulin resistance - a state of impaired peripheral tissue response to insulin; it depends on both genetic and environmental conditions - peripheral tissues, which may occur several years before the onset of fully symptomatic diabetes. Elevated glucose levels are observed when this 
compensatory ability of $\beta$ cells becomes depleted and there is a relative deficiency of insulin.

The most characteristic feature of patients with type 2 diabetes is overweight and obesity, as well as: age above 50 years of age, history of diabetes in the family; low physical activity; recurrent infection (especially of the skin and urinary tract) in the history; features of glucose intolerance observed in the past; abdominal obesity; other features of the metabolic syndrome: hypertension, lipid disorders; existing vascular complications (heart attack, stroke, retinopathy, nephropathy); moderately elevated, high or very high $\mathrm{HbA}_{1 c}$ value at the time of diagnosis [13-15].

Subjective symptoms are non-specific and varied, related to the type of diabetes and the dynamics of the disease. More than half of the cases of type 2 diabetes are asymptomatic, but also lead to the development of chronic complications, especially cardiovascular ones, which are the main cause of death in these patients [4]. Clinically, in patients with type 2 diabetes, good glycaemic response to increased physical activity and dietary restrictions, as well as to oral drugs, is observed in the early stages of the disease $[16,17]$.

\section{Gestational diabetes}

Gestational diabetes mellitus (GDM) is diagnosed as having hyperglycaemia during pregnancy in a woman who has not yet had diabetes mellitus, with values corresponding to the diagnosis of diabetes mellitus or any of the conditions of glucose intolerance [17]. During pregnancy, especially in the 2nd and 3rd trimesters, there is a physiological decrease in insulin sensitivity and a worsening of glucose tolerance [18].

The groups of increased risk of developing diabetes during pregnancy are: elderly women; obese women; women with glycosuria (presence of glucose in urine) found in two accidentally diagnosed tests during pregnancy; pregnant women with hydramnios; women with glucose intolerance in history; history of birth of a newborn with bodyweight $>4.5 \mathrm{~kg}$; in family history — first-degree relatives diabetes mellitus.

\section{Life-threatening conditions resulting from complications of diabetes mellitus}

\section{Diabetic ketoacidosis}

Diabetic ketoacidosis is an acute complication of diabetes, which is a direct life-threatening condition.
Untreated ketoacidosis can lead to diabetic coma and even death. The main causes of diabetic ketoacidosis include discontinuation of insulin therapy or making some mistakes, e.g. inappropriate doses; late diagnosis of type 1 diabetes; alcohol abuse; acute inflammations (e.g. bacterial, viral, fungal infections); and pregnancy. The occurrence of ketoacidosis may be directly life-threatening. The most severe (although rare) complication may be cerebral oedema, which is more common in children. In addition, hypovolemic shock and acute renal failure may also occur. The cause of the disorders is a significant deficiency of insulin leading to hyperglycaemia, reduced sensitivity to insulin in conditions of systemic infection or an increase in the concentration of hyperglycaemic hormones. Insulin deficiency leads to the release of free fatty acids and their subsequent oxidation in the liver to ketone compounds. Glycosuria and hyperglycaemia lead to osmotic diuresis, dehydration and electrolyte disorders.

In case of diabetic ketoacidosis, the patient may present the following symptoms: abdominal pain, which is most common in children, headaches, excessive fatigue and general weakness, deepened and accelerated breathing (the so-called Kussmaul breathing); specific mouth and urine acetone smell similar to the smell of rotten apples as well as consciousness disturbances. Symptoms of ketoacidosis may be similar to those of poisoning or gastrointestinal infections. If elevated blood glucose levels persist for a prolonged period, especially if vomiting occurs, the presence of acetone in urine or blood ketone levels should be checked by means of special test strips.

When differentiating between diabetic ketoacidosis, one should consider disorders such as "acute abdomen", hyperglycaemic conditions with different pathogenesis, including hyperglycaemic and hyperosmolar conditions, as well as coma of other origins such as hypoglycaemic, uremic, hepatic, cerebral and resulting from poisoning.

Emergency management in diabetic ketoacidosis is based on securing basic vital functions according to $A B C D E$ scheme. The next element is the supplementation of fluid deficits in the vascular bed. In order to do this, it is necessary to start with $1 \mathrm{~L}$ infusion of isotonic solution $0.9 \% \mathrm{NaCl}$ within the first hour of treatment. The remaining deficit, which usually ranges from 5 to $10 \mathrm{~L}$, should be corrected within 6 to 12 hours. The administration of short-acting insulin in a single dose of $8-10$ units is another 
step in the management. A continuous infusion at $0.1 \mathrm{unit} / \mathrm{kg}$ body weight per hour is then administered. The dose should be adjusted individually to lowered glycaemia with a rate of $50-70 \mathrm{mg} / \mathrm{dL} / \mathrm{hr}$. If glycaemia is lowered below $200 \mathrm{mg} / \mathrm{dL}$, an infusion of $5 \%$ glucose solution should be implemented. The dose of potassium used should be $20 \mathrm{mEq}$ of potassium chloride per litre of fluid for patients with normokaliemia and $40 \mathrm{mEq} / \mathrm{L}$ for patients with hypokalemia.

\section{Hyperglycaemic-hyperosmolar state - HHS}

Hyperglycaemic-hyperosmolar state is a metabolic disorder that occurs with significant dehydration of the body as a result of disturbed mechanisms regulating thirst or limited fluid intake. The hyperglycaemic-hyperosmolar state results from the symptoms of uncontrolled or untreated diabetes. Blood glucose increases first, usually exceeds $600 \mathrm{mg} / \mathrm{dL}$ (33.3 mmol/L) [19]. As a consequence, osmotic diuresis, systemic electrolyte deficiencies and dehydration occur. Then the hyperosmolarity of the serum, usually above $350 \mathrm{mmol} / \mathrm{L}$, occurs with simultaneously observed symptoms of ketoacidosis and metabolic acidosis.

The most common causes of hyperglycaemic-hyperosmolar state include pneumonia, cardiovascular disorders, kidney failure, urinary tract infection, acute pancreatitis or excessive alcohol consumption. It may also result from iatrogenic complications, including diuretics treatment or peritoneal dialysis.

A patient with hyperglycaemic-hyperosmolar state requires treatment in an intensive care unit. The symptoms of HHS usually develop gradually - within a few days, contrary to diabetic ketoacidosis - where the symptoms develop within a few hours [20]. The symptoms associated with significant dehydration appear: dry mucosa, loss of skin elasticity, increased thirst. In addition, one observes tachypnea well as tachycardia, reduced blood pressure and headaches. Depending on the severity of developed HHS, there are variously intense consciousness disorders, including coma. Sometimes seizures, paresis of extremities, visual disturbances can be observed.

The differential diagnosis of hyperglycaemic-hyperosmolar state should include acute disorders of the central nervous system, coma resulting from poisoning or coma of other origins, including hypoglycaemic, liver, brain, uremic or ketone disorders $[21,22]$.
The treatment is multidirectional, the most important is hydration, intravenous insulin administration, infection control and treatment of coexisting diseases. However, from the point of view of emergency medicine, management should include restoring normal fluid volume and compensating for electrolyte deficiencies [23]. For this purpose, consider the infusion of $1-2 \mathrm{~L}$ of $0.9 \%$ physiological $\mathrm{NaCl}$ solution within the first hour of treatment. It is worth remembering that half of the fluid deficit should be supplemented within 12 hours. Another aspect is potassium deficiency compensation. The dose of potassium should be $20 \mathrm{mEq}$ of potassium chloride per litre of fluid for patients with normokalemia and $40 \mathrm{mEg} / \mathrm{L}$ for patients with hypokalemia. Insulin therapy is another element of emergency management. Treatment should begin with a single dose of 8-10 units of intravenous short-term insulin, followed by an infusion at 0.1 unit/kg body weight per hour. The dose should be adjusted individually to reduce glycaemia at a rate of $50-70 \mathrm{mg} / \mathrm{dL} / \mathrm{h}$. If glycaemia is reduced below $200 \mathrm{mg} / \mathrm{dL}$, an infusion of $5 \%$ glucose solution should be implemented [24].

\section{Hypoglycaemia}

Hypoglycaemia is the most common complication in diabetic patients treated with insulin or oral hypoglycaemic drugs [25]. Hypoglycaemia may also occur, apart from the two factors mentioned above, as a result of a delayed meal or glucose products intake, physical activity, excessive calorie loss, alcohol consumption or excessive glucose consumption and impairment of its use in gluconeogenesis [26]. An American Diabetes Association (ADA) working party has attempted a comprehensive definition [27]. According to this association, "severe hypoglycaemia" is defined as an episode requiring the assistance of another person to administer treatment, "documented symptomatic hypoglycaemia" as the presence of common symptoms of hypoglycaemia with a measured plasma glucose $<3.9 \mathrm{mmol} / \mathrm{L}$, "probable symptomatic hypoglycaemia" as self-reported symptoms not verified by a glucose measurement and "relative hypoglycaemia" as the presence of symptoms with a plasma glucose $>3.9 \mathrm{mmol} / \mathrm{L}$.

While in the case of hyperglycaemia it takes a relatively long time for the patient to develop consciousness disorders, in the case of hypoglycaemia these changes occur quickly. In most cases, a patient can feel that his blood glucose level decreases, but in case of adrenergic reactions, increased expression 


\begin{tabular}{|c|c|}
\hline Blood glucose level & Symptoms \\
\hline $\begin{array}{l}\text { Mild } \\
\text { hypoglycaemia }\end{array}$ & $\begin{array}{l}\text { Hunger, sweat, pale skin, anxiety, } \\
\text { tachycardia, paraesthesia }\end{array}$ \\
\hline $\begin{array}{l}\text { Moderate } \\
\text { hypoglycaemia }\end{array}$ & $\begin{array}{l}\text { Headaches, drowsiness, blurred } \\
\text { vision, concentration disorders, } \\
\text { aggressive behaviour }\end{array}$ \\
\hline $\begin{array}{l}\text { Severe } \\
\text { hypoglycaemia }\end{array}$ & $\begin{array}{l}\text { Hyperventilation, paresis, tonic and } \\
\text { clonic seizures, confusion and then } \\
\text { a loss of consciousness, bilateral } \\
\text { symptom of Babinski, initially increased } \\
\text { reflexes disappear completely and } \\
\text { muscles become flaccid }\end{array}$ \\
\hline
\end{tabular}

of glucotransporter genes or neuropathy - the feeling of increasing symptoms of hypoglycaemia may be significantly impaired [27].

Depending on the severity of the hypoglycaemia, we can expect an adequate patient's condition [28, 29]. A detailed summary of the symptoms of hypoglycaemia by blood glucose level is given in Table 1 [30].

During the hypoglycaemic state, the body begins to release so-called counterregulatory hormones, which are intended to counteract the growing hypoglycaemia. These hormones include catecholamine, glucagon, growth hormone and cortisol [31-33].

The causes of hypoglycaemia could be numerous. However, in the case of diabetics, a decrease in blood glucose level is most often a consequence of overdose of insulin or sulfonylurea derivatives a stimulant of its secretion [34].

During differentiation, many potential pathologies and causes of hypoglycaemia should be considered, including epileptic seizure, stroke, alcohol abuse, depression, psychosis, dehydration and electrolyte disorders, liver or kidney failure or overdose of sympatheticomimetric drugs.

The management of hypoglycaemia in a patient who is conscious and presents symptoms of mild to moderate hypoglycaemia should be directed to oral administration of simple sugars first and then complex sugars which will be released longer. In case of unconscious patients, in addition to protection of the airway, monitoring and intravenous access, intravenous glucose $20 \% / 5 \%$ at a dose of $0.2 \mathrm{~g} / \mathrm{kg}$ body weight should be administered intravenously until a blood concentration of $100 \mathrm{mg} / \mathrm{dL}$ is achieved. If intravenous access is not available, $1 \mathrm{mg}$ of glucagon should be administered intramuscularly (Fig. 1), to increase the release of glucose from the liver. It should be remembered, however, that the

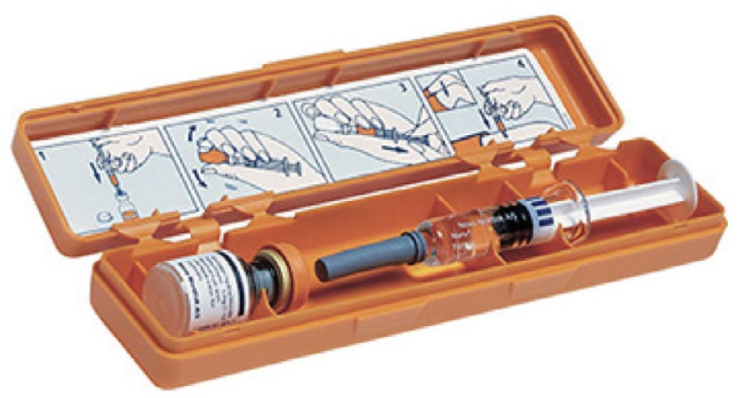

FIGURE 1. A pre-filled syringe with a glucagon

glucagon's action is limited or completely eliminated in case of liver tissue damage, prolonged hunger strike or alcohol abuse [35]. In the latter two cases, an additional $100 \mathrm{mg}$ of thiamine should be considered, and in the case of prolonged hypoglycaemia, hydrocortisone may be necessary to counteract adrenal insufficiency. Regardless of whether we lead to euglycemia or not, the patient should be transported to the hospital.

\section{Lactic acidosis}

Lactic acidosis, besides ketoacidosis, diabetic hyperosmolar syndrome or hypoglycaemia is one of the acute complications of diabetes. It occurs as a result of intensification of anaerobic glucose metabolism. Although it is rare, it is characterized by a high mortality rate of $25-50 \%$.

Lactic Acidosis is not a specific complication only for diabetes. There are many reasons for this condition. This condition is characterized by an increase in blood lactate levels above $5 \mathrm{mmol} / \mathrm{L}$ and a simultaneous decrease in $\mathrm{pH}$ below 7.35. It develops mainly in type 2 diabetes as a result of biguanide treatment and does not take into account contraindications - especially renal failure - and tissue hypoxemia due to advanced lung diseases or atherosclerosis. In type 1 diabetes, especially when there are renal failure and microangiopathy, lactate acidosis may be a complication of severe ketoacidosis with high dehydration and shock symptoms. Currently, due to the cause of lactate acidosis, it is divided into type $A$ and type $B$. Type $A$ is acidosis formed in anaerobic conditions as a result of heart failure, respiratory failure or shock including hypovolemic, cardiogenic or septic shock. In the case of type B lactate acidosis, it is formed independently of tissue hypoxia and occurs as a result of liver diseases - which metabolize lactic acid to pyruvic acid, kidney diseases — which excrete lactic acid along with urine, as well as may 
be associated with severe complications of diabetes, including ketoacidosis, or may be the result of improper use of metformin, abuse of methyl or ethyl alcohol or high doses of salicylates [36].

In diabetes, lactate acidosis concerns mainly patients treated with metformin. The term Metformin-Associated Lactic Acidosis (MALA) refers to any case of lactate acidosis that has developed in methamphetamine treated patients [37]. It is noteworthy that MALA occurs when metformin is accumulated in the body under the influence of triggering factors, including tissue hypoxemia and renal failure [38]. The exact mechanism, as well as the role of metformin in MALA formation, is not finally explained. Lactic acid is the final product of anaerobic glucose metabolism and occurs mainly in skeletal muscles and erythrocytes. The lactate is then transported to the liver, where it is oxidised to pyruvate and finally converted by gluconeogenesis into glucose in the so-called Cori cycle [39] (Fig. 2). Inhibition of mitochondrial chain and decrease in cellular production of ATP reduces the level of hepatic gluconeogenesis and causes increased concentration of lactic acid, which is a specific substrate of retained gluconeogenesis. Increased lactic acid levels may also increase the transition from aerobic to anaerobic metabolism [40]. Lactic acid accumulated in the serum in this way is a consequence of the occurrence of acidosis.

During an in-depth medical history the patient usually complains about gastrointestinal problems such as nausea, vomiting, abdominal pain or diarrhoea. Clinical examination shows signs of dehydration and exhaustion. Kussmaul's breathing (deep and fast), thready and rapid pulse and reduced blood pressure are observed. As a result of the above symptoms, there is a reduced cardiac index and hypotension, which manifests itself in the form of disorders of consciousness and circulatory failure leading to shock, coma or death. Clinical symptoms may resemble myocardial infarction, heavy bleeding

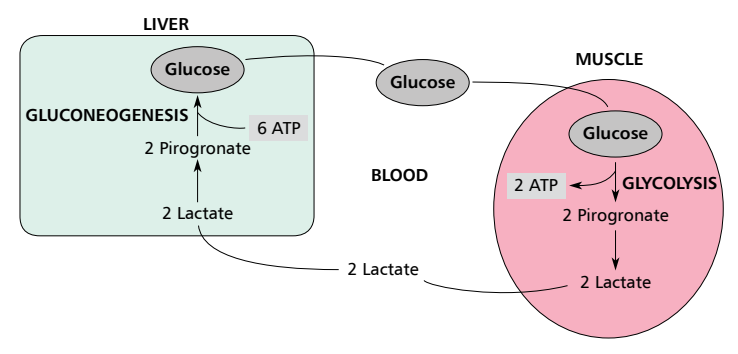

FIGURE 2. Developed on the basis of Cori Cycle or pneumonia, as they are the specific initiators of lactate acidosis.

In the differential diagnosis it is worth to take into account other aetiologies of acidosis, including ketoacidosis or hyperglycaemic-hyperosmolar acidosis. In the case of patients in coma, other causes of coma, including ketones, hypoglycaemic, cerebral, hepatic or toxic coma, should be considered [41-43].

Treatment consists primarily in causative treatment (withdrawal of the drug in case of suspicion of its correlation with lactate acidosis [40]) and anti-shock management, including: oxygen therapy, hydration, intravenous infusion of catecholamines, or reduction of hypoglycaemia and acidosis by infusion of insulin at a rate of 0.1 unit $/ \mathrm{kg}$ body weight per hour in an appropriate combination with 5\% glucose solution. In exceptional cases - at pH below 7.0 - sodium bicarbonate may also be considered - however, it should be noted that in such a case the patient should be properly ventilated, because although sodium bicarbonate reduces respiratory acidosis, in case of inadequate ventilation the patient may exacerbate intracellular acidosis.

\section{CONCLUSIONS}

Due to the frequency of life-threatening complications, diabetes is a heavy burden for the medical staff. Adequate diagnosis and implementation of appropriate treatment significantly improve the patient's condition.

\section{REFERENCES}

1. Tuchman AM. History of diabetes. MD Advis. 2013; 6(1): 8-13, indexed in Pubmed: 24013300.

2. Maahs DM, West NA, Lawrence JM, et al. Epidemiology of type 1 diabetes. Endocrinol Metab Clin North Am. 2010; 39(3): 481-497, doi: 10.1016/j.ecl.2010.05.011, indexed in Pubmed: 20723815.

3. de Ferranti SD, de Boer IH, Fonseca V, et al. Type 1 diabetes mellitus and cardiovascular disease: a scientific statement from the American Heart Association and American Diabetes Association. Circulation. 2014; 130(13): 1110-1130, doi: 10.1161/CIR.0000000000000034, indexed in Pubmed: 25114208.

4. Pozzilli P, Pieralice S. Latent Autoimmune Diabetes in Adults: Current Status and New Horizons. Endocrinol Metab (Seoul). 2018; 33(2): 147-159, doi: 10.3803/EnM.2018.33.2.147, indexed in Pubmed: 29947172.

5. Li Q, Qiao ZR, Liu DB, et al. Relationship between serum GAD-Ab and the genetic polymorphisms of GAD2 and type 2 diabetes mellitus. Genet Mol Res. 2015; 14(2): 3002-3009, doi: 10.4238/2015. April.10.10, indexed in Pubmed: 25966064. 
6. Suzuki R. T-cell function in anti-GAD65 + diabetes with residual $\beta$-cell function. Journal of Autoimmunity. 2003; 20(1): 83-90, doi: 10.1016/ s0896-8411(02)00093-8.

7. Becerril Ángeles M, García Lara SE, González Bárcena D, et al. [Autoantibodies in GAD65 in Mexican adults with diabetes types 1 and 2 and their siblings]. Rev Alerg Mex. 2010; 57(6): 190-195, indexed in Pubmed: 21944486.

8. Moloney TC, Idris I, Waters $P$, et al. Autoantibodies to glutamic acid decarboxylase in patients with epilepsy and their relationship with type 1 diabetes: a pilot study. J Neurol Neurosurg Psychiatry. 2016; 87(6): 676677, doi: 10.1136/jnnp-2015-310512, indexed in Pubmed: 25995487.

9. Harris AG, Letourneau LR, Greeley SA. Monogenic diabetes: the impact of making the right diagnosis. Curr Opin Pediatr. 2018; 30(4): 558-567, doi: 10.1097/MOP.0000000000000643, indexed in Pubmed: 29846255.

10. Sanyoura M, Philipson LH, Naylor R. Monogenic Diabetes in Children and Adolescents: Recognition and Treatment Options. Curr Diab Rep. 2018; 18(8): 58, doi: 10.1007/s11892-018-1024-2, indexed in Pubmed: 29931562.

11. Mishra R, Hodge KM, Cousminer DL, et al. A Global Perspective of Latent Autoimmune Diabetes in Adults. Trends Endocrinol Metab. 2018; 29(9): 638-650, doi: 10.1016/j.tem.2018.07.001, indexed in Pubmed: 30041834.

12. DeFronzo RA. Pathogenesis of type 2 diabetes mellitus. Med Clin North Am. 2004; 88(4): 787-835, ix, doi: 10.1016/j.mcna.2004.04.013, indexed in Pubmed: 15308380.

13. Yazdanpanah $S$, Rabiee $M$, Tahriri $M$, et al. Evaluation of glycated albumin $(\mathrm{GA})$ and $\mathrm{GA} / \mathrm{HbA} 1 \mathrm{c}$ ratio for diagnosis of diabetes and glycemic control: A comprehensive review. Crit Rev Clin Lab Sci. 2017; 54(4): 219-232, doi: 10.1080/10408363.2017.1299684, indexed in Pubmed: 28393586.

14. Fletcher $B$, Gulanick M, Lamendola C. Risk factors for type 2 diabetes mellitus. J Cardiovasc Nurs. 2002; 16(2): 17-23, indexed in Pubmed: 11800065.

15. Whitmore C. Type 2 diabetes and obesity in adults. Br J Nurs. 2010; 19(14): 880, 882-880, 886, doi: 10.12968/bjon.2010.19.14.49041, indexed in Pubmed: 20647979.

16. Caruso R, Magon A, Baroni I, et al. Health literacy in type 2 diabetes patients: a systematic review of systematic reviews. Acta Diabetol. 2018; 55(1): 1-12, doi: 10.1007/s00592-017-1071-1, indexed in Pubmed: 29129000.

17. Chiefari E, Arcidiacono B, Foti D, et al. Gestational diabetes mellitus: an updated overview. J Endocrinol Invest. 2017; 40(9): 899-909, doi: 10.1007/s40618-016-0607-5, indexed in Pubmed: 28283913.

18. Ananthakrishnan S. Diabetes insipidus during pregnancy. Best Practice \& Research Clinical Endocrinology \& Metabolism. 2016; 30(2): 305-315, doi: 10.1016/j.beem.2016.02.005.

19. Stoner GD, Stoner GD. Hyperosmolar hyperglycemic state. Am Fam Physician. 2005; 71(9): 1723-1730, indexed in Pubmed: 15887451.

20. Scott AR. Joint British Diabetes Societies (JBDS) for Inpatient Care, JBDS hyperosmolar hyperglycaemic guidelines group. Management of hyperosmolar hyperglycaemic state in adults with diabetes. Diabet Med. 2015; 32(6): 714-724, doi: 10.1111/dme.12757, indexed in Pubmed: 25980647.

21. Fayfman M, Pasquel FJ, Umpierrez GE. Management of Hyperglycemic Crises: Diabetic Ketoacidosis and Hyperglycemic Hyperosmolar State. Med Clin North Am. 2017; 101(3): 587-606, doi: 10.1016/j. mcna.2016.12.011, indexed in Pubmed: 28372715.

22. Frank LA, Solomon A. Hyperglycaemic hyperosmolar state. $\mathrm{Br}$ J Hosp Med (Lond). 2016; 77(9): C130-C133, doi: 10.12968/ hmed.2016.77.9.C130, indexed in Pubmed: 27640667.

23. Yared Z, Chiasson JL. Ketoacidosis and the hyperosmolar hyperglycemic state in adult diabetic patients. Diagnosis and treatment. Minerva Med. 2003; 94(6): 409-418, indexed in Pubmed: 14976469.

24. Umpierrez G, Korytkowski M. Diabetic emergencies - ketoacidosis, hyperglycaemic hyperosmolar state and hypoglycaemia. Nat Rev Endocrinol. 2016; 12(4): 222-232, doi: 10.1038/nrendo.2016.15, indexed in Pubmed: 26893262.

25. Giménez-Cassina A, Garcia-Haro L, Choi CS, et al. Regulation of hepatic energy metabolism and gluconeogenesis by BAD. Cell Metab. 2014; 19(2): 272-284, doi: 10.1016/j.cmet.2013.12.001, indexed in Pubmed: 24506868.

26. Workgroup on Hypoglycemia, American Diabetes Association. Defining and reporting hypoglycemia in diabetes: a report from the American Diabetes Association Workgroup on Hypoglycemia. Diabetes Care. 2005; 28(5): 1245-1249, doi: 10.2337/diacare.28.5.1245, indexed in Pubmed: 15855602.

27. Ebadi SA, Darvish P, Fard AJ, et al. Hypoglycemia and cognitive function in diabetic patients. Diabetes Metab Syndr. 2018; 12(6): 893-896, doi: 10.1016/j.dsx.2018.05.011, indexed in Pubmed: 29887517.

28. Rossi MC, Nicolucci A, Ozzello A, et al. HYPOS-1 Study Group of AMD. Impact of severe and symptomatic hypoglycemia on quality of life and fear of hypoglycemia in type 1 and type 2 diabetes. Results of the Hypos-1 observational study. Nutr Metab Cardiovasc Dis. 2019; 29(7): 736-743, doi: 10.1016/j.numecd.2019.04.009, indexed in Pubmed: 31153746.

29. Musen G, Jacobson AM, Ryan CM, et al. Diabetes Control and Complications Trial/Epidemiology of Diabetes Interventions and Complications Research Group. Impact of diabetes and its treatment on cognitive function among adolescents who participated in the Diabetes Control and Complications Trial. Diabetes Care. 2008; 31(10): 1933-1938, doi: 10.2337/dc08-0607, indexed in Pubmed: 18606979.

30. Shaefer C, Hinnen D, Sadler C. Hypoglycemia and diabetes: increased need for awareness. Curr Med Res Opin. 2016; 32(9): 1479-1486, doi: 10.1185/03007995.2016.1163255, indexed in Pubmed: 26959277.

31. Lee $Y H$, Wang MY, Yu XX, et al. Glucagon is the key factor in the development of diabetes. Diabetologia. 2016; 59(7): 1372-1375, doi: 10.1007/s00125-016-3965-9, indexed in Pubmed: 27115412.

32. Williams KM, Fazzio P, Oberfield SE, et al. Cortisol Levels in Children With Diabetic Ketoacidosis Associated With New-Onset Type 1 Diabetes Mellitus. Clin Pediatr (Phila). 2017; 56(2): 117-122, doi: 10.1177/0009922816684595, indexed in Pubmed: 28145127. 
33. Feldman JM, Plonk JW, Bivens CH. The role of cortisol and growth hormone in the counter-regulation of insulin-induced hypoglycemia. Horm Metab Res. 1975; 7(5): 378-381, doi: 10.1055/s-0028-1093731, indexed in Pubmed: 1183916.

34. Thulé PM, Umpierrez G. Sulfonylureas: a new look at old therapy. Curr Diab Rep. 2014; 14(4): 473, doi: 10.1007/s11892-014-0473-5, indexed in Pubmed: 24563333.

35. Arky RA. Hypoglycemia associated with liver disease and ethanol. Endocrinol Metab Clin North Am. 1989; 18(1): 75-90, indexed in Pubmed: 2645130.

36. Claudino WM, Dias A, Tse W, et al. Type B lactic acidosis: a rare but life threatening hematologic emergency. A case illustration and brief review. Am J Blood Res. 2015; 5(1): 25-29, indexed in Pubmed: 26171281.

37. Rüegg T, Caduff B. Accumulation of Metformin-associated Lactic Acidosis. Dtsch Med Wochenschr. 2017; 142(6): 428-431, doi: 10.1055/s-0042-119280, indexed in Pubmed: 28329904.

38. Hevesy MR. Metformin-Associated Lactic Acidosis: An Atypical Presentation. Adv Emerg Nurs J. 2017; 39(1): 26-30, doi: 10.1097/ TME.0000000000000134, indexed in Pubmed: 28141608.
39. Rubin RP. Carl and Gerty Cori: A collaboration that changed the face of biochemistry. J Med Biogr. 2019 [Epub ahead of print]: 967772019866954, doi: 10.1177/0967772019866954, indexed in Pubmed: 31475888.

40. Moioli A, Maresca B, Manzione A, et al. Metformin associated lactic acidosis (MALA): clinical profiling and management. J Nephrol. 2016; 29(6): 783-789, doi: 10.1007/s40620-016-0267-8, indexed in Pubmed: 26800971.

41. Bianchetti DG, Amelio GS, Lava SAG, et al. D-lactic acidosis in humans: systematic literature review. Pediatr Nephrol. 2018; 33(4): 673-681, doi: 10.1007/s00467-017-3844-8, indexed in Pubmed: 29218437.

42. Schuh AM, Leger KJ, Summers C, et al. Lactic Acidosis in a Critically III Patient: Not Always Sepsis. Pediatr Emerg Care. 2018; 34(9): e165-e167, doi: 10.1097/PEC.0000000000001603, indexed in Pubmed: 30180106.

43. Halperin ML. Lactic acidosis and ketoacidosis: biochemical and clinical implications. Can Med Assoc J. 1977; 116(9): 1034-1038, indexed in Pubmed: 404010. 